\title{
Strength Model Evaluation Based on Experimental Measurements of Necking Profile in Ductile Metals
}

\author{
Lorenzo Peroni ${ }^{1}$, Martina Scapin ${ }^{1, *}$ \\ ${ }^{1}$ Politecnico di Torino, Department of Mechanical and Aerospace Engineering, Corso Duca degli Abruzzi 24, 10129 Turin, Italy
}

\begin{abstract}
The reliability of the numerical results strongly depends upon the choice of the constitutive models, which sometimes requires an ad-hoc calibration of the parameters starting from experimental results. Often, the tension test is chosen since it allows obtaining a big amount of data, but in case of ductile materials, in which the specimen could be subjected to high value of plastic strain before the fracture, it is necessary to properly manage the experimental results. For low values of deformation, the deformation of the gage length of the specimen is uniform and the true stress can be easily derived from the longitudinal one and considered as the equivalent stress. At the onset of necking, geometrical instability and concentration of deformation are developed. In the scientific literature several different approaches have been proposed in order to estimate the equivalent stress distribution in the post-necking regime. In this work, a new approach was proposed by the combination of digital image analysis and numerical inverse method. The evolution of the profile deformation of the specimen observed during the test is directly used to identify the constitutive relation of the material in case of dynamic tensile tests.
\end{abstract}

\section{Introduction}

Nowadays, the finite element simulations are a valid and widespread tool for the design phase in several fields as well as in the understanding of complex phenomenon allowing, in some cases, the limitation of full-scale experimental tests, which could be dangerous and expensive. Nevertheless, the reliability of the numerical results strongly depends upon the choice of the constitutive models chosen as well as the failure one. In the actual paper the attention is focused on the determination of the plastic flow rule which sometimes requires an ad-hoc calibration of the parameters starting from experimental results. Often, the tension test is chosen since it allows obtaining a big amount of data, both on plastic flow stress and failure. Anyway, in case of ductile materials, in which the specimen could be subjected to high value of plastic strain before the fracture, it is necessary to properly manage the experimental results. In case of ductile metals and alloys, for which large strains can be reached before fracture, the data analysis at the basis of identification process has to be carefully treated. At small strains (in elastic and nearly plastic regime), the specimen deforms homogenously both along cross section and gage length.

The longitudinal strain can be calculated, using strain-gages, extensometer or digital image correlation, as the engineering one (ratio between the stroke and the initial gage length) or as the logarithmic one. The value obtained is equal to the equivalent strain. Accordingly, also the uniaxial stress can be derived and used as the equivalent stress. After the maximum of the engineering stress is reached, the presence of geometrical instability and the localization of necking produce a complex triaxial state of stress characterized by large strains. In this regime, the longitudinal strain has to be actualized with respect to the portion of the specimen in which the deformation is localized: the estimation of the effective strain can be performed by measuring the actual diameter of the minimum cross section. On the other hand, the determination of the equivalent stress, which is no longer equal to the uniaxial one, is more complicate. With the aim to take into consideration also the postnecking behaviour, several methods were developed. A first well-known solution was based on the Bridgman's theory and its following formulation [1-2]. Other widespread approaches were correlated to the measurement of the strain fields inside the neck and based on the balance between external and internal works (e.g. Virtual Field Methods) [3-4]. Another possibility is represented by numerical inverse methods based on FE simulations, in which, independently from the strain measurement, the flow stress identification was performed on the iterative matching between macroscopic quantities (e.g. force vs. displacement curve) [5-6]. In any case, it is important to consider that to perform the model calibration based on stress (i.e. force) or strain can produce different or inconsistent results depending on the adopted strength model.

For this reason, the main goal of the present paper is to propose an alternative technique able to joint stress and strain information during the model calibration procedure. In the scientific literature in this direction, some proposals were done, but in which basically the

Corresponding author: martina.scapin@polito.it 
force information was added to the strain full-field measurement [7-8]. In this paper a different approach is proposed since the same technique has to be used also for high temperature condition, in which it is not possible to make and follow a speckle on the specimen surface, hence it is not possible to apply DIC techniques. As final consideration, the proposed methodology could be used to identify the material flow stress in postnecking regime and by varying the temperature and strain-rate conditions.

\section{The necking profile}

The method here proposed combines the information coming from the measurement of the necking profile with the information about the force time history (or force-stroke signal), in order to extract the flow stress curve as a function of plastic strain, strain-rate and temperature starting from tensile tests. The method consists on the flow stress calibration via an iterative procedure based on FE simulations, which reproduce the same loading conditions of the experimental tests. It can be applied to different loading conditions in terms of strain-rate and temperature, since the only requirement for the application of the method is to record the images of the necking profile variation during the tests and to precisely synchronize them with the force signal.

By considering dog-bone specimens with round cross-section (fig. 1) the measurement of the radial displacement gives the information of the complete strain tensor at the external surface. As a matter of fact, the specimen surface is in a plane stress state, since the radial stress is zero. Moreover, due to the fact that the longitudinal stress is bigger in comparison to the circumferential (hoop) one at the surface, the radial strain can be approximate with the circumferential one, which can be directly obtained by the radial displacement. In case of small deformation the relationship between circumferential strain and radial displacement $u$ is $\varepsilon_{\mathrm{c}}=u / r$, which becomes $\varepsilon_{\mathrm{c}}=\ln (1+u / r)$ in case of high deformations. Under the hypothesis of volume conservation, the longitudinal strain is equal to two times the circumferential one (opposite sign).

At a certain level of deformation after necking, the shape of the specimen is similar to that reported in fig. 1. As already discussed, each cross-section is deformed in function of the radial displacement $u$. Points with a greater radial displacement exhibit a greater level of strain and consequently strain-rate. The reduction in cross-section produces an increase in the level of the axial stress: along the specimen, each point is characterized by a unique combination of equivalent stress, strain and strain-rate and linked to the neighbourhood points with a certain hardening-rate $\mathrm{d} \sigma / \mathrm{d} \varepsilon$. In case of adiabatic test also the temperature varies along the sample in relation to the amount of plastic work.

These considerations are useful to understand why it is possible to consider the external shape of the sample (e.g. the deformed profile) as a direct consequence of the flow stress curve of the material. As a matter of fact, there is a one-to-one correspondence between the twos: a fixed flow stress curve produces a specific deformed shape of the sample and a certain deformed shape is possible only with a particular flow stress curve. Actually, the same specimen profile can be obtained from scaled flow stress curves (e.g. it is equivalent to a change in the units system of the stress definition): hence, it is necessary an additional information regarding the level of stress to get a unique flow stress curve.

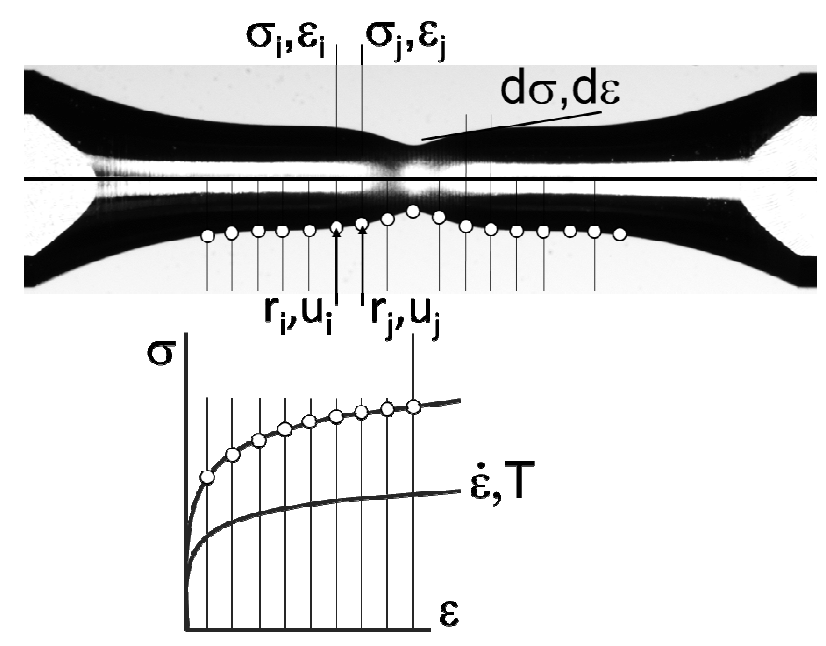

Fig. 1. Specimen profile after necking: correlation between the shape and the flow stress curve.

A problem is related to the choice of the flow stress model to be used to predict the experimental response. If the model is able to reproduce the correct material response in terms of shape of the sample, then the corresponding stress-strain curve is the correct one: this is true if the material model is able to correctly take into account all the variables of the problem, like strain, strain-rate and temperature. Obtaining the right shape means that each point of the specimen surface follows the right path in terms of strain, strain-rate and temperature and consequently is associated to the right value of stress. In the methodology here proposed, the shape of the specimen could be experimentally evaluated, hence, the measurement of the strain-state is quite precise and consequently also the evaluation of the strain-rate is possible with high accuracy. On the other hand, the measurement of the temperature is very difficult (even if not impossible but quite expensive and complicate [9]) so it is necessary to adopt a numerical estimation based on the conversion of the plastic work with the Taylor-Quinney approach [10] and this aspect will be discussed in the following.

Depending on the loading condition in terms of both strain-rate and temperature, a material could exhibit different necking profiles: if the loading condition produces a global strain hardening (i.e. strain-rate increment or temperature decrease), the necking profile involves a wide portion of the specimen; on the contrary, if the loading condition produces a strain softening (i.e. high temperature), the necking profile is characterized by a narrow portion of the sample with a strong localization 
of strain, strain-rate and temperature in the necking zone [11].

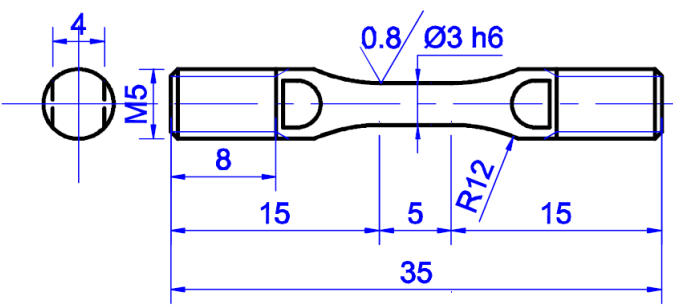

Fig. 2. Sketch of the cylindrical dog bone specimen.

It is possible to apply this procedure, based on the experimental measurement of the radial displacement is to a material in which the effects of strain-rate and temperature are negligible on the mechanical behaviour or for a loading condition such that the material is loaded in an isothermal way with a negligible effect of strain rate. To test the proposed approach a benchmark case was developed by creating an ad-hoc experimental profile obtained via a numerical simulation of a sample (fig. 2) with a known J-C material model (the reference hardening parameters are: $A=446 \mathrm{MPa}, B=511 \mathrm{MPa}$ and $n=0.221$ [5]). The experimental test was simulated and the shape of the specimen and the load-stroke curve of the sample obtained from the numerical model. The FE model of the dog-bone specimen was solved in LSDYNA and built by 2D-axisymmetric shell elements with 1 integration point (fig. 3).

A symmetric velocity profile was applied to the two specimen heads, which has to be consistent with the experimental one. To the central part of the specimen a re-meshing algorithm was applied in order to avoid too high element deformation and distortion during the postnecking deformation: as a consequence, the element characteristic dimension was kept as constant as possible. The computed quantities which were extracted for the optimization procedure were: the longitudinal force vs. imposed displacement and the radial displacements of the nodes on the external surface. The longitudinal force vs. displacement curve is reported in fig. 3 , in which also the sequence of the deformed profile is reported. Each marker in the diagram corresponds to one of the model images, from which it is possible to appreciate the effect of the re-meshing algorithm, which allows describing the necking zone in a reliable way up to high deformation level.

\section{The optimization procedure}

At this stage the FE model was implemented in the optimization software LS-OPT, with the aim to get the correct flow stress via the iterative procedure. The profile of the sample was defined as objective function and used by the optimization algorithm to manage the change of the strength model parameters: 41 points along the necking profile were monitored in order to check the sample shape. To fix the scale factor of the flow stress curve, a single point in load history was used: since the correct prediction of the necking onset is an important issue in a tool based on the necking profile, the chosen point is the maximum of load-stroke curve in order to correctly impose the strain at which there is the instability onset as well as the corresponding level of stress.
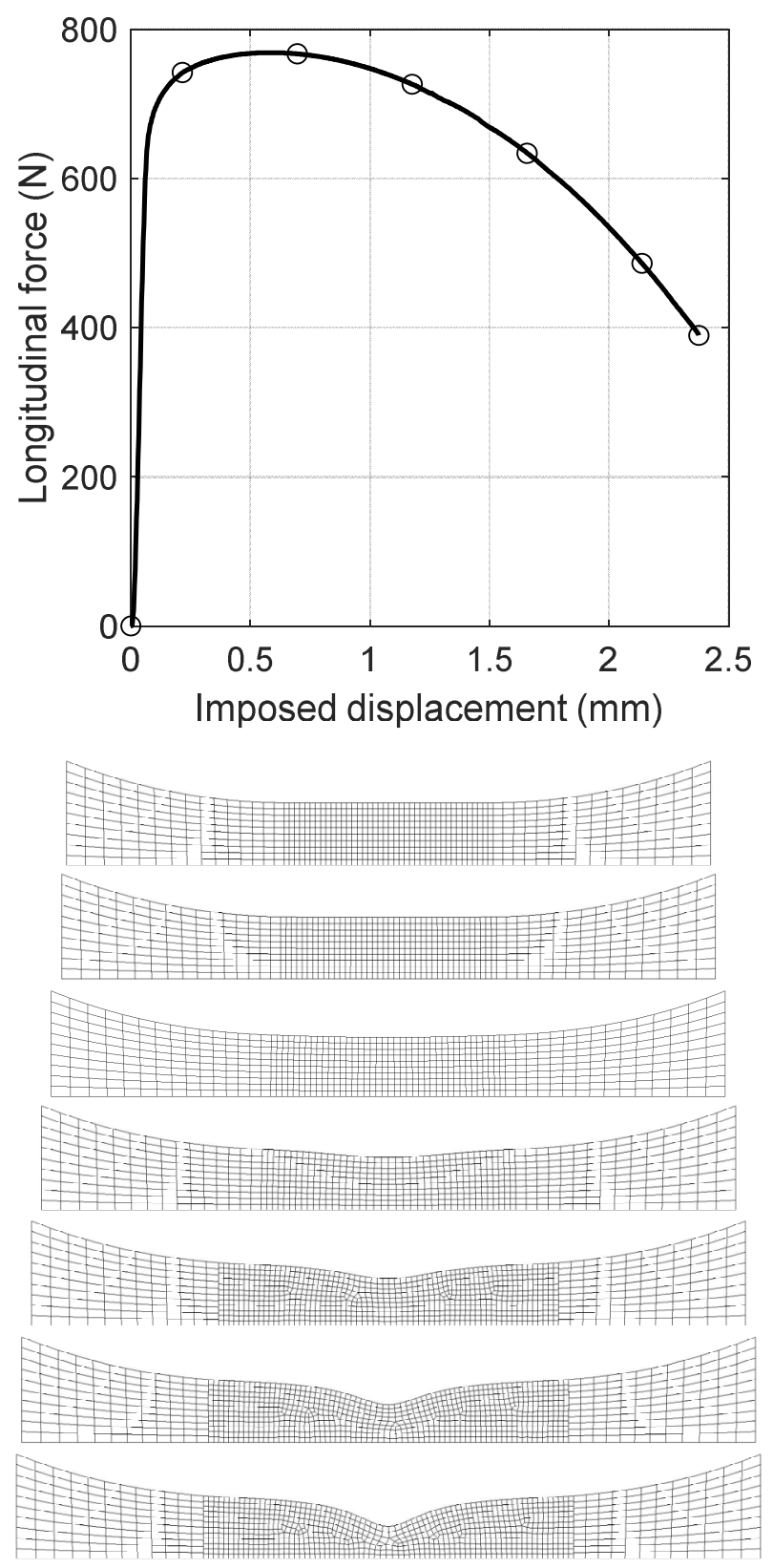

Fig. 3. Computed force vs. displacement curve; sequence of deformed profile images.

Two different approaches could be followed. The first one implies the simultaneous use of a series of specimen profiles at different levels of deformation up to high level of deformation. The second one implies the use of a single post-necking specimen profile at a high level of deformation. Greater the reached level of deformation, wider the range of strain in which the strength model is evaluated (as previously discussed the maximum plastic equivalent strain is easily predictable by the maximum radial displacement). In a real experimental case, the last deformed profile used for the 
optimization must be sufficiently far from the initiation of the ductile damage.

With these two objective functions (maximum load and specimen shape) the optimization tool finds a solution very closed to the right one in both the approaches. The Root Mean Square Error (RMSE) was used to evaluate the differences between the expected and the computed values both in term of stress and profile. For the case in which a series of 10 profiles were used, for a plastic strain range varying between 0 and 1 , the RMSE in terms of stress was $10 \mathrm{MPa}$, while a RMSE of about $2 \mu \mathrm{m}$ was found in terms of profile. For the case in which a just the last of the 10 profiles was used, the RMSE in terms of stress was 3.5 MPa, while a RMSE of about $1 \mu \mathrm{m}$ was found in terms of profile. Since both the solutions are acceptable, it could be more convenient to adopt the solution with a unique profile. This approach has also the advantage to give the possibility to get a high-resolution image even at high strain-rate (low frame-rate is necessary) or the interesting possibility to use a post-test image of the recovered from interrupted test.

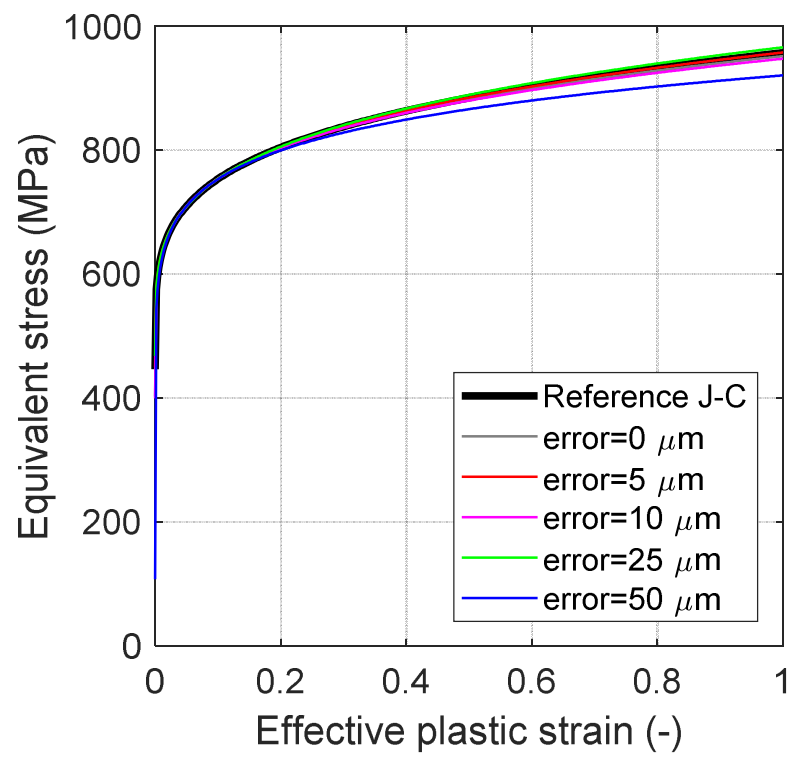

Fig. 4. Comparison in terms of J-C flow stress curves obtained from the optimization procedure by varying the imposed error on the necking profile.

To test the robustness of the approach, a noise was added to the ad-hoc created shape profile to investigate the effects of possible errors in the experimental measurement of the profile. To this aim, a random uniform noise, with different probability density factors, was added to the reference profile: a minimum error of $5 \mu \mathrm{m}$ and a maximum one of $50 \mu \mathrm{m}$ were considered. The corresponding profiles used as targets in the optimization procedure are reported in fig. 6 .

By considering the profile corresponding to the maximum error, it is possible to conclude that, for sure, it represents a worst case in comparison to the capability in the profile measurement from experimental video recorded images (see for comparison the quality of the image reported in fig. 1 and in [12]). Moreover, it has to be considered that an experimental measurement of the profile if too noised can be interpolated in order to reduce the level of noise and to smooth the profile by neglecting non-physical high frequency oscillations. Moreover, if the recorded image is of the full sample, 4 equivalent semi-profile are measured for each test and an average low-noise profile could be obtained.

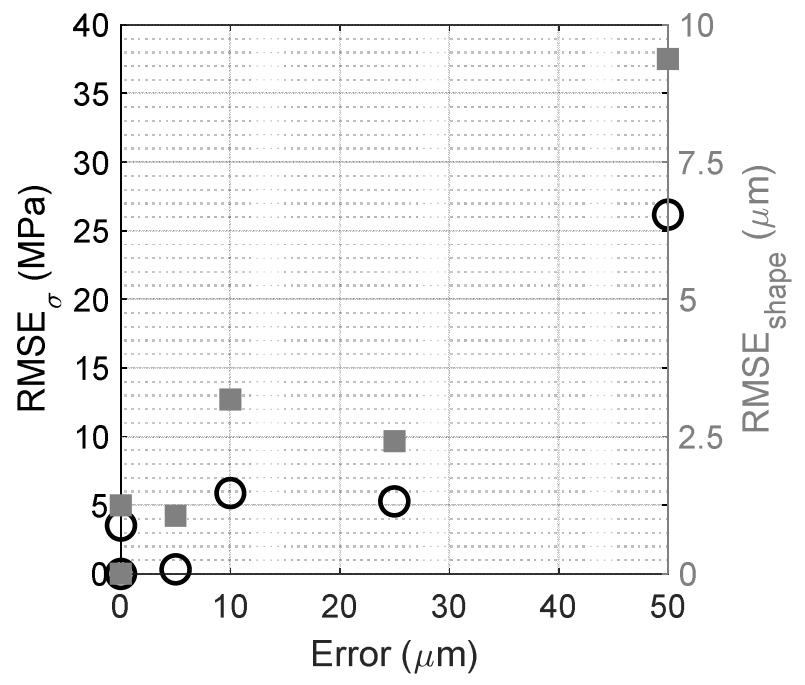

Fig. 5. RMSE for stress and shape obtained by varying the imposed error on the necking profile.

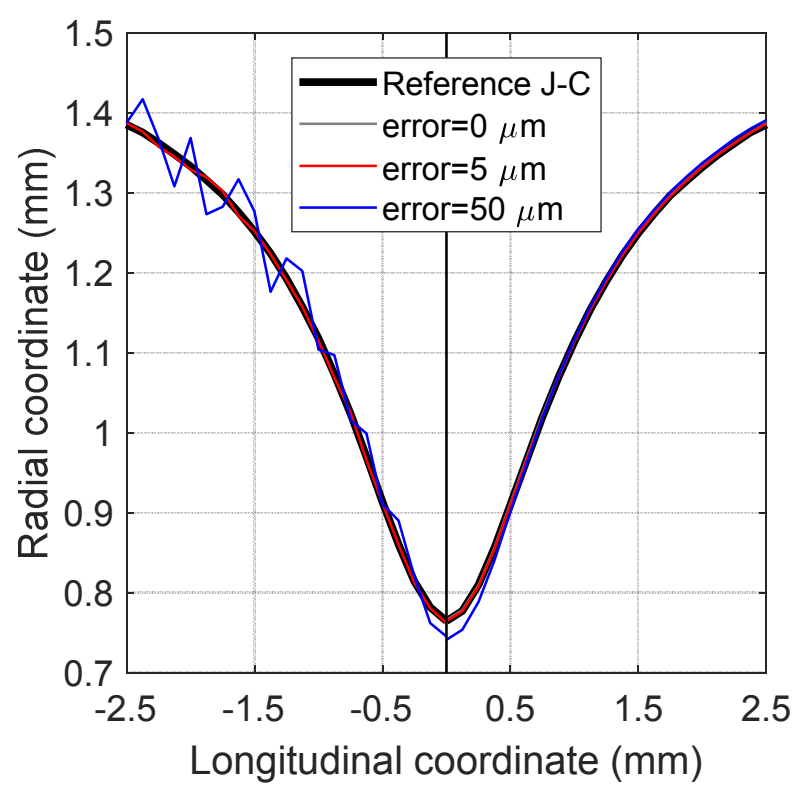

Fig. 6. Comparison between the imposed target (left side) and the optimized profile (right side) for some values of the imposed error on the necking profile with respect to the reference shape.

The results of the optimizations for different levels of noise are reported in fig. 5 in terms of RMSE and in fig. 6 in terms of profile: the procedure seems to be sufficiently robust also for high level of noise since the maximum RMSE in stress is less than $30 \mathrm{MPa}$ and the maximum RMSE in profile is less than $10 \mu \mathrm{m}$. The comparison in terms of optimized J-C flow stress curves is reported in fig. 4: by looking at the diagram it is possible to conclude that for a level of noise up to 
$25 \mu \mathrm{m}$, the optimized curves cannot be distinguished from the reference one, especially at low strain. This is a further prove of the capability of the procedure to find the correct solution.

\section{Dynamic loading conditions}

To extend this approach for the identification of strength model in dynamic loading condition the optimization tool must be able to correctly attribute the effects of the increase in strength due to the strain-rate increase along the sample and the effect of the strength reduction due to the adiabatic temperature increment along the sample (fig. 1). A possible strategy is to perform a multi-case optimization, in which the algorithm has to simultaneously satisfy the multiple objectives coming from different loading conditions: a dynamic (adiabatic) test, a quasi-static (isothermal) test and a dynamic (adiabatic) test at a different starting temperature. In this way the local effects of temperature and strain-rate changes in the sample has to be consistent with the effect of moving the reference condition of the same quantities.

As performed for the previous case, even in this one an ad-hoc benchmark case was created, by considering the complete J-C formulation implemented in LS-DYNA (the total set of parameters are: $A=446 \mathrm{MPa}$, $B=511 \mathrm{MPa}, n=0.221, \mathrm{C}=0.0219, \dot{\varepsilon}=0.001 \mathrm{~s}^{-1}, m=1.35$, $\left.T_{m}=1142 \mathrm{~K}, T_{r}=298 \mathrm{~K}[5]\right)$.

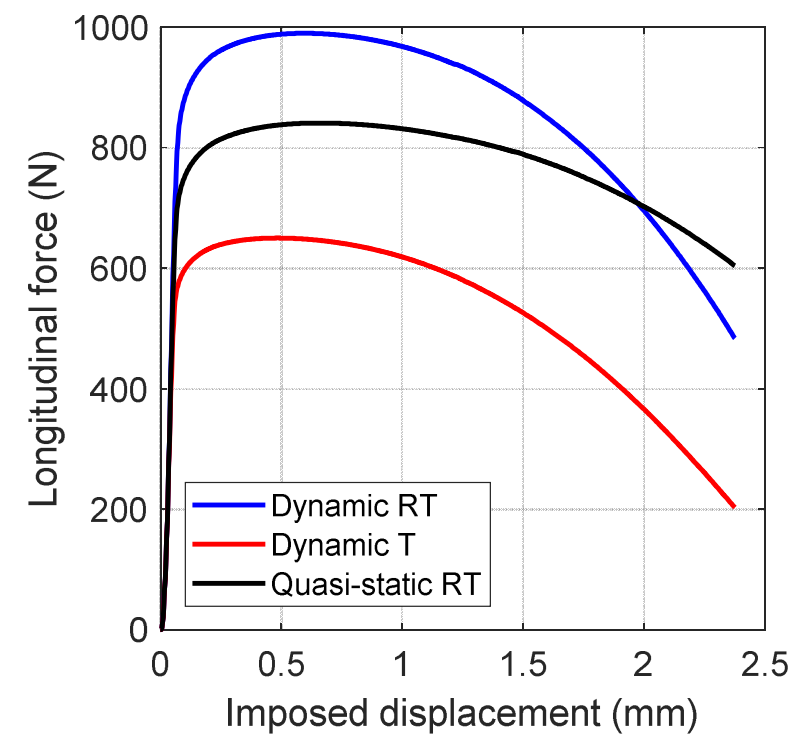

Fig. 7. Computed force vs. displacement curve for the loading conditions used in the multi-case optimization.

The three different chosen cases correspond respectively to: a dynamic tensile test performed at room temperature (RT) at a nominal strain-rate of $1000 \mathrm{~s}^{-1}$, a dynamic tensile test performed at $673 \mathrm{~K}$ (which corresponds to the maximum temperature increment due to adiabatic heating of the previous loading condition) and at a nominal strain-rate of $1000 \mathrm{~s}^{-1}$, end finally a quasi-static tensile test performed at room temperature at a nominal strain-rate of $0.1 \mathrm{~s}^{-1}$ (which was chosen as the highest strain-rate for which the deformation could be assumed isothermal). Each case was simulated and the specimen profile associated with the corresponding loadstroke curve was obtained from the numerical model. In all the model the same final displacement was imposed, even if it could not be the same in a real experimental campaign [5]: the value was chosen in order to overcome the $100 \%$ of effective plastic strain in the dynamic case at room temperature.

The diagram of fig. 7 shows the computed longitudinal force vs. longitudinal imposed displacement for the three cases. No noise was applied to the specimen profiles. The same optimization procedure used for the single-case optimization was applied to extract the best set of material model parameters. For each loading condition, the objective functions were a unique profile in the post-necking regime (monitored in 41 points) and the maximum value of the longitudinal force vs. displacement curve.

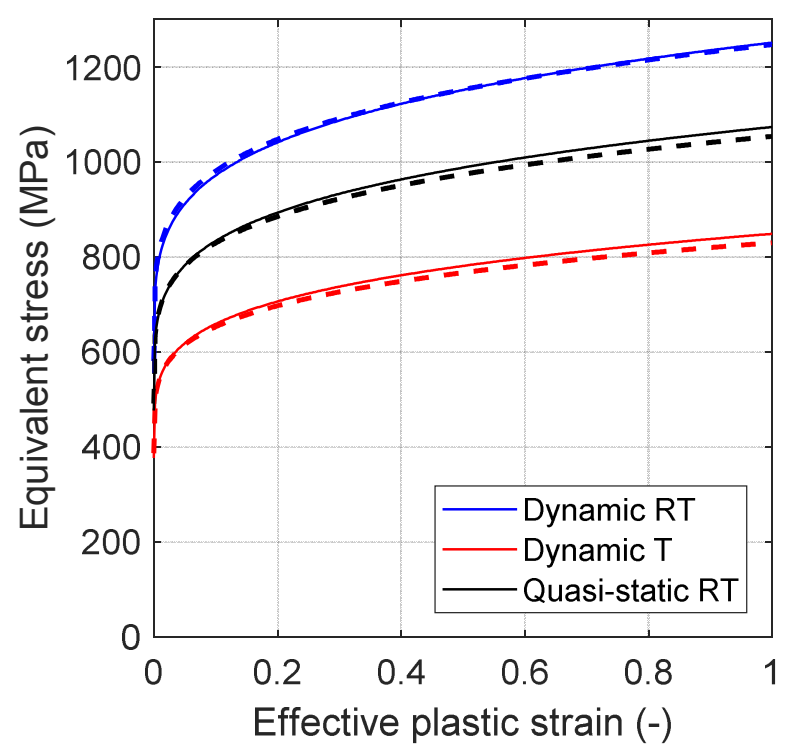

Fig. 8. Comparison in terms of J-C flow stress curves obtained from the optimization procedure for different loading conditions.

In the diagram of fig. 8 , the comparison between the expected J-C flow stress curves (dashed lines) and those obtained by using the optimized set of parameters (solid lines) is reported. The three curves represent the reference conditions of the considered loading condition: neither variation of temperature nor strain-rate was taken into account. Obviously since each point of the specimens experiences a specific path on the surface defined by strain, strain-rate and temperature, this comparison is just qualitative. As a matter of fact, in fig. 9, the spatial distributions along the specimen of equivalent plastic strain, strain-rate, temperature and Von Mises stress were reported for the dynamic loading condition at room temperature. The images are referred to the same time in which the objective functions in terms of radial displacements were defined. As it is possible to notice were the strain reaches the maximum value $(130 \%)$, the temperature increase is about $350{ }^{\circ} \mathrm{C}$ and the strain-rate is 7 times the nominal value $\left(1000 \mathrm{~s}^{-1}\right)$. 


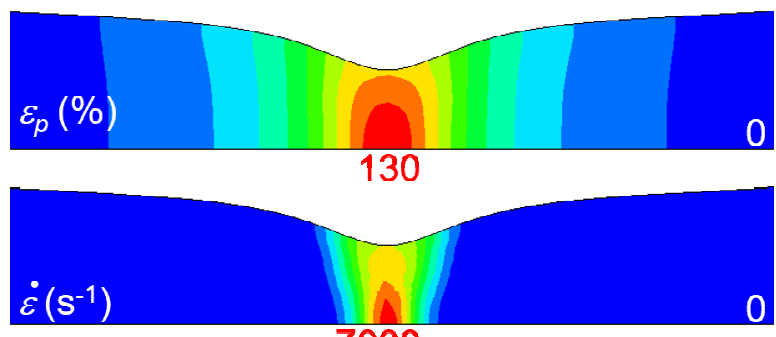

7000
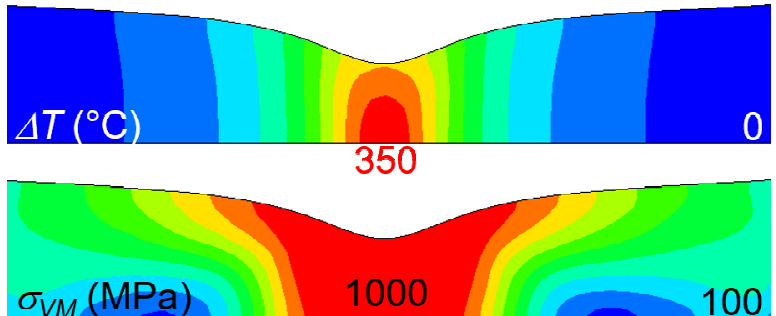

Fig. 9. Spatial distribution along the specimen of equivalent plastic strain, strain-rate, temperature and Von Mises stress.

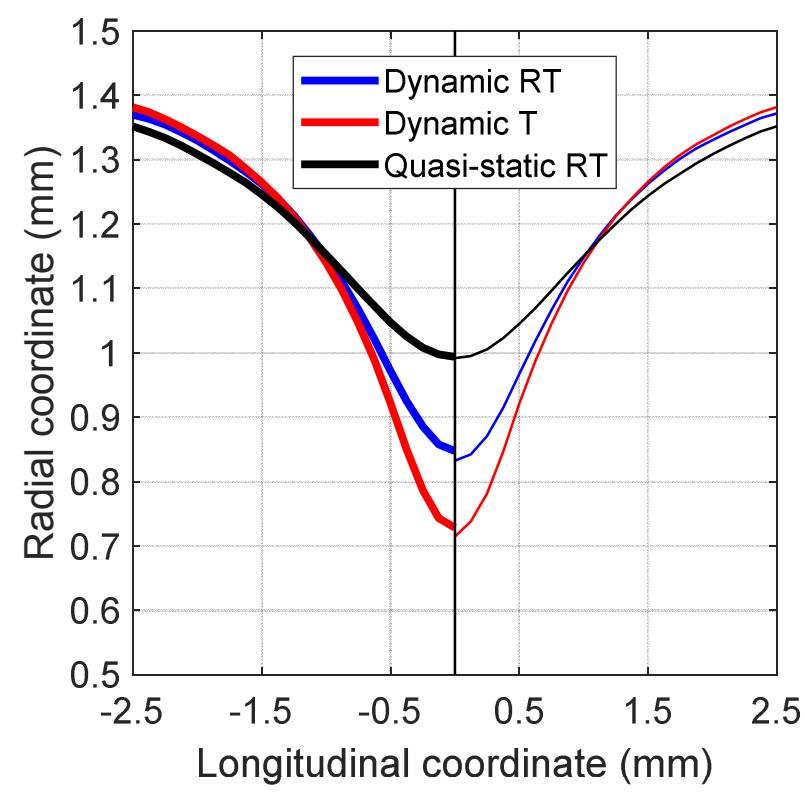

Fig. 10. Comparison between the imposed target (left side) and the optimized profile (right side) for different loading conditions.

A more quantitative evaluation of the capability of the optimized J-C model to correctly predict the material response was obtained by calculating the RMSE in a lot of possible configurations by simultaneously varying also strain-rate and temperature in addition to strain. The ranges of variation of the variables were decided based on the minimum and maximum expected values with respect to the reference ones. In this condition a RMSE of about $11 \mathrm{MPa}$ was found which was correlated to an average RMSE on the profiles of about $4 \mu \mathrm{m}$ for the three loading conditions. The comparison in terms of necking profile was reported in fig. 10. The results in terms of RMSE both in terms of stress and profile showed that, even in this case, the procedure is able to find a flow stress curve very closed to the expected one. In this case in which the experimental data were replaced by ad-hoc numerical targets (without noise), the differences between the obtained parameters and the reference ones are only due to the optimization algorithm and its capacity of finding the right solution respect to other equivalent ones. The precision could be certainly improved by changing the type or some parameters of the optimization software.

\section{Conclusions}

In this paper an approach to identify post-necking flow stress curve by also including strain-rate and temperature effects starting from tensile test results was discussed. Differently from more commonly used methodologies, the optimization was performed by simultaneously considering stress and strain objective functions. The idea at the basis of the work is that the specimen profile, measured after the onset of necking, includes all the necessary information to completely define the strain state of the specimen surface. The strain distribution along the specimen could be obtained by the measurement of the radial displacement: this data, combined with the maximum load value (necking onset) and used as objective function and in a numerical optimization procedure allows the identification of the parameters of a thermo-visco-plastic material model like Johnson-Cook one.

\section{References}

1. P.W. Bridgman, Studies in large plastic flow and fracture (McGraw-Hill, New York, 1952)

2. J. Aronofskt, J. Appl. Mech. Trans. ASME, 18, 1 (1951)

3. F. Pierron, S. Zhavoronok, M. Grédiac, Int. J. Solids Struct. 37, 2 (2000)

4. S. Coppetiers, S. Cooreman, H. Sol, P. Van Houtte, D. Debruyne, J. Mater. Process. Technol. 211, 3 (2011)

5. M. Scapin, L. Peroni, C. Fichera, A. Cambriani, J. Mater. Eng. Perform. 23 (2014)

6. J. Peirs, P. Verleysen, W. Van Paepegem, J. Degrick, Int. J. Impact Eng. 38, 5 (2011)

7. J. Kajberg, G. Lindkvist, Int. J. Solids Struct. 41, 3 (2004)

8. A. J. Gross, K. Ravi-Chandar, J. Appl. Mech. Trans. ASME 82, 7 (2015)

9. D. Rittel, L. H. Zhang, S. Osovski, J. Mech. Phys. Solids 107 (2017)

10. J. D. Seidt, V. T. Kuokkala, J. L. Smith, A. Gilat, Exp. Mech. (2016)

11. M. Scapin, L. Peroni, F. Carra, JDBM 2, 4 (2016)

12. M. Scapin et al., Int. J. Imp. Eng. 106 (2017) 